\title{
ON INTERPOLATION of FUNCTIONS with a BOUNDARY LAYER BY CUBIC SPLINES
}

\author{
I.A. Blatov ${ }^{1}$, E.V.Kitaeva ${ }^{1}$, A.I. Zadorin ${ }^{2}$ \\ ${ }^{1}$ Volga Region State University of Telecommunications and Informatics, Samara, Russia \\ ${ }^{2}$ Sobolev Institute of Mathematics of Siberian Branch of Russian Academy of Sciences, \\ Novosibirsk, Russia
}

\begin{abstract}
The problem of article is cubic spline-interpolation of functions having high gradient regions. It is shown that uniform grids are inefficient to be used. In case of piecewise-uniform grids, concentrated in the boundary layer, for cubic spline interpolation are announced asymptotically exact estimates on a class of functions with an exponential boundary layer. There are obtained results showing divergent in small parameter estimates and divergence of interpolation processes. The modified cubic spline with uniform in small parameter interpolation error is offered. The results of numerical experiments confirming theoretical estimates are given.
\end{abstract}

Keywords: boundary layer, singular perturbation, cubic spline-interpolation, error estimates.

Citation: Blatov IA, Kitaeva EV, Zadorin AI. About interpolation by cubic splines of the functions with a boundary layers. CEUR Workshop Proceedings, 2016; 1638: 515-520. DOI: 10.18287/1613-0073-2016-1638-515-520

\section{Introduction}

Cubic splines are widely applied to smooth interpolation of functions. Such splines are investigated in [1], [2] and in many other works. However, according to [3], [4], application of the polynomial spline-interpolation to functions with large gradients in a boundary layer leads to essential errors of $O(1)$ type. In [4] there was constructed a non-polynomial analogue of cubic spline, which is exact for the boundary layer component. Numerical experiments have shown the advantage in the accuracy of the constructed spline. However, the boundary layer component isn't always known, thus, in such case, there is no reasonable alternative for condensing a grid in the boundary layer. In this work a traditional cubic spline interpolation [2] on the piecewise uniform grid condensing in the boundary layer is investigated. There were obtained error estimates of interpolation which, however, aren't uniform in small parameter $\varepsilon$. It is shown that an interpolation error of a boundary layer component might increase without limits while $\varepsilon \rightarrow 0$, thus, we need to develop special methods of interpolation for such functions. Such a method of interpolation is also offered and investigated in 
this work. We shall pass it noting that the divergence of interpolation processes by cubic and parabolic splines on nonuniform grids was regarded in works [2], [5], [6] and some other works. However, the examples of divergence provided there had an artificial character or were implied with the help of Banach-Steinhaus theorem. In the present article we have shown divergence for functions describing solutions of a variety of applied problems. These results testify the need of development of universal high-order methods of smooth spline interpolation of functions on nonuniform grids and development of projective-grid methods of a high order for singular perturbed boundary value problems, because there is no need for application of the grid solution interpolation while using projective-grid methods.

\section{Statement of the problem}

Let us introduce the following notations. Let $\Omega: 0=x_{0}<x_{1}<\cdots<x_{N}=1-$ partition of a segment $[0,1]$. Let $S(\Omega, k, 1)$ be a space of polynomial splines [2] of degree $k$ and defect 1 on the grid $\Omega$. We means that $C, C_{j}$ are positive constants independent from $\varepsilon$ and a number of grid nodes. We write $f=O(g)$ if $|f| \leq C|g|$ and $f=O^{*}(g)$, if $f=O(g)$ and $g=O(f), C[a, b]$ is a space of continuous functions with norm $\|\cdot\|_{C[a, b]}$.

Let a function $u(x)$ have a form

$u(x)=q(x)+\Phi(x), x \in[0,1]$,

$\left|q^{(j)}(x)\right| \leq C_{1},\left|\Phi^{(j)}(x)\right| \leq C_{1} e^{-\alpha x / \varepsilon} / \varepsilon^{j}, 0 \leq j \leq 4$.

Let us investigate a problem of cubic spline-interpolation of the function (1).

\section{Main results}

First, let us look into the case of a uniform grid. Let $N$ be a natural number, $\Delta$ is uniform grid with a step of $H=1 / N$ and nodes $x_{n}, n=0,1, \cdots, N$, partitioning of the interval $[0,1]$. Let $g_{3}(x, u) \in S(\Delta, 3,1)$ be an interpolation cubic spline on the grid $\Delta$, defined by conditions:

$$
g_{3}\left(x_{n}, u\right)=u\left(x_{n}\right), 0 \leq n \leq N, g_{3}{ }^{\prime}(0, u)=u^{\prime}(0), g_{3}{ }^{\prime}(1, u)=u^{\prime}(1) \text {. }
$$

Theorem 1. In case of a uniform grid there shall be such a constant $C$ for which the next estimate is correct:

$\left\|u(x)-g_{3}(x, u)\right\|_{C[0,1]} \leq C(N \varepsilon)^{-4}$.

If in (1) $\Phi(x)=e^{-\alpha x / \varepsilon}$, then the following estimate holds: 
$\left\|u(x)-g_{3}(x, u)\right\|_{C[0,1]} \geq C_{1} \min \left\{(N \varepsilon)^{-1},(N \varepsilon)^{-4}\right\}$.

Next, according to [7] let us define the grid $\Omega$ with nodes $x_{n}, n=0,1, \cdots, N$, and steps

$$
h_{n}=h=\frac{\sigma}{N / 2}, n=1, \cdots, \frac{N}{2}, h_{n}=H=\frac{1-\sigma}{N / 2}, n=\frac{N}{2}+1, \cdots, N .
$$

In accordance with [7] let us define

$$
\sigma=\min \left\{\frac{1}{2}, \frac{4 \varepsilon}{\alpha} \ln N\right\} \text {. }
$$

Due to [2] for interpolation cubic spline $g_{3}(x, u) \in S(\Omega, 3,1)$ the following error estimate is valid:

$$
\left|g_{3}(x, u)-u(x)\right| \leq \frac{5}{384}\left\|u^{(4)}\right\|_{C[0,1]} \max _{n} h_{n}^{4} .
$$

Note that $g_{3}(x, u)=g_{3}(x, q)+g_{3}(x, \Phi)$, and due to (1),(4)

$$
\left\|q(x)-g_{3}(x, q)\right\|_{C[0,1]} \leq C_{2} \max _{n} h_{n}^{4} \leq C_{2} N^{-4} \text {. }
$$

Henceforth, in order to build a spline interpolation to $u(x)$ with the order of $O\left(N^{-4} \ln ^{4} N\right)$, it is needed to satisfy the inequality:

$\left\|\Phi(x)-g_{3}(x, \Phi)\right\|_{C[0,1]} \leq C_{2} N^{-4} \ln ^{4} N$.

In case when in (5) $\sigma=1 / 2$, an inequality (4) shall be valid in account of Theorem 1 and due to the relation $N \varepsilon=O^{*}(N / \ln N)$. Thus, we shall propose below that $\sigma<1 / 2$. Yet, to keep it short we shall assign $g_{3}(x)=g_{3}(x, \Phi)$, $g_{3}(x) \in S(\Omega, 3,1)$.

Theorem 2. There are such constants $C_{2}, C_{3}$, which satisfy the relation (5) if $N^{-1} \leq C_{3} \varepsilon$.

Theorem 3. There are such constants $C_{4}, C_{5}$, and $\beta>0$, independent from $\varepsilon, N$, that if $\varepsilon \leq C_{4} N^{-1}$, then

$$
\left\|g_{3}(x, \Phi)-\Phi(x)\right\|_{C\left[x_{n}, x_{n+1}\right]} \leq C_{5}\left\{\begin{array}{c}
N^{-4} \ln ^{4} N, 0 \leq n \leq N / 2-1 \\
\frac{N^{-5}}{\varepsilon} e^{-\beta(n-N / 2)}, N / 2 \leq n \leq N-1
\end{array} .\right.
$$

Next Theorem shows us that estimates in (6) can not be improved. 
Theorem 4. Let $\Phi(x)=e^{-x / \varepsilon}$.Then there are such constants $C_{4}, C_{6}, \beta_{1}>0$, independent from $\varepsilon, N$ that if $\varepsilon \leq C_{4} N^{-1}$, then

$\left\|g_{3}(x, \Phi)-\Phi(x)\right\|_{C\left[x_{n}, x_{n+1}\right]} \geq C_{6} \frac{N^{-5}}{\varepsilon} e^{-\beta_{1}(n-N / 2)}, \frac{N}{2} \leq n \leq N-1$

Now we shall construct a modified interpolation spline. Let $\bar{x}_{N / 2}=\left(x_{N / 2}+x_{N / 2+1}\right) / 2, \quad \bar{x}_{n}=x_{n}, \quad n \in[0, N / 2-1] \cup[N / 2+1, N]$. Let $g m_{3}(x, u)$ be the interpolation cubic spline defined by conditions $g m_{3}\left(\bar{x}_{n}, u\right)=u\left(\bar{x}_{n}\right), n \in[0, N], g m_{3}{ }^{\prime}(0, u)=u^{\prime}(0), g m_{3}{ }^{\prime}(1, u)=u^{\prime}(1)$.

The only difference between $g m_{3}(x, u)$ and $g_{3}(x, u)$ is that the interpolation node $x_{N / 2}$ is set as $\bar{x}_{N / 2}$. The spline nodes whereas are not subject to any changes and coincide with the $\Omega$ nodes.

Theorem 5. There are such independent from $\varepsilon, N$ constants, namely $\gamma_{0}>0, C$ that if $\varepsilon \ln N \leq \gamma_{0}$, it shall satisfy the following inequality:

$\left\|u(x)-g m_{3}(x, u)\right\|_{C[0,1]} \leq C N^{-4} \ln ^{4} N$.

Comment 1. The condition $\varepsilon \ln N \leq \gamma_{0}$ will be satisfied if $\varepsilon \leq C N^{-1}$.

Therefore, according to theorems 2,5 application of the interpolation spline $g m_{3}(x, u)$ if $\varepsilon=O\left(N^{-1}\right)$ and the interpolation spline $g_{3}(x, u)$ if $N^{-1}=O(\varepsilon)$ let as to obtain estimates (4), (7) uniformly in $\varepsilon, N$.

\section{Results of Numerical Experiments}

Let us define the following function:

$u(x)=\cos \frac{\pi x}{2}+e^{-\frac{x}{\varepsilon}}, x \in[0,1]$

Results of calculations are provided in the three following tables. Given in the tables below are the maximum errors of spline interpolation, calculated at nodes of the condensed grid, which is obtained from the initial grid by splitting every single grid interval into 10 parts. Table 1 contains interpolation errors for the traditional cubic spline on the uniform grid. Results confirm estimates of the Theorem 1 and an inadequacy of application of the uniform grid for small values of $\varepsilon$. Table 2 provides errors of a traditional cubic spline in Shishkin meshes. It follows from the tables that errors increase while $\varepsilon$ decreases and $N$ is fixed. Results of Table 3 describing the 
errors of modified cubic spline, on the contrary, show uniform convergence, thus, theoretical conclusions are confirmed.

Table 1. Errors of cubic spline on the uniform grid

\begin{tabular}{lllllll}
\hline \multicolumn{1}{c}{$\mathbf{N}$} & $\mathbf{1 6}$ & $\mathbf{3 2}$ & $\mathbf{6 4}$ & $\mathbf{1 2 8}$ & $\mathbf{2 5 6}$ & $\mathbf{5 1 2}$ \\
$\boldsymbol{\varepsilon}$ & & & & & & \\
\hline $\mathbf{1}$ & $2.82 \mathrm{e}-7$ & $1.76 \mathrm{e}-8$ & $1.16 \mathrm{e}-9$ & $1.02 \mathrm{e}-10$ & $4.30 \mathrm{e}-11$ & $\mathbf{2 . 6 8 e}-\mathbf{1 3}$ \\
$\mathbf{1 0 e}-\mathbf{1}$ & $3.43 \mathrm{e}-4$ & $2.33 \mathrm{e}-5$ & $1.51 \mathrm{e}-6$ & $9.58 \mathrm{e}-8$ & $6.03 \mathrm{e}-9$ & $\mathbf{4 . 1 1 e}-\mathbf{1 0}$ \\
$\mathbf{1 0 e}-\mathbf{2}$ & 0.43 & $8.38 \mathrm{e}-2$ & $9.72 \mathrm{e}-2$ & $8.00 \mathrm{e}-4$ & $5.59 \mathrm{e}-5$ & $\mathbf{3 . 6 5 e}-\mathbf{6}$ \\
$\mathbf{1 0 e}-\mathbf{3}$ & 9.88 & 4.58 & 1.93 & 0.66 & 0.15 & $\mathbf{2 . 0 3 e - 2}$ \\
$\mathbf{1 0 e}-\mathbf{4}$ & $1.05 \mathrm{e}+2$ & $5.23 \mathrm{e}+1$ & $2.58 \mathrm{e}+1$ & $1.25 \mathrm{e}+1$ & 5.90 & $\mathbf{2 . 5 9}$ \\
$\mathbf{1 0 e}-\mathbf{5}$ & $1.06 \mathrm{e}+3$ & $5.23 \mathrm{e}+2$ & $2.64 \mathrm{e}+2$ & $1.32 \mathrm{e}+2$ & $6.56 \mathrm{e}+1$ & $\mathbf{3 . 2 4 e + 1}$ \\
$\mathbf{1 0 e}-\mathbf{6}$ & $1.06 \mathrm{e}+4$ & $5.30 \mathrm{e}+3$ & $2.65 \mathrm{e}+3$ & $1.33 \mathrm{e}+3$ & $6.62 \mathrm{e}+2$ & $\mathbf{3 . 3 0 e}+\mathbf{2}$ \\
$\mathbf{1 0 e}-\mathbf{7}$ & $1.06 \mathrm{e}+5$ & $5.30 \mathrm{e}+4$ & $2.65 \mathrm{e}+4$ & $1.33 \mathrm{e}+4$ & $6.63 \mathrm{e}+3$ & $\mathbf{3 . 3 0 e + 3}$ \\
\hline $\mathbf{1 0 e}-\mathbf{8}$ & $\mathbf{1 . 0 6 e}+\mathbf{6}$ & $\mathbf{5 . 3 0 e}+\mathbf{5}$ & $\mathbf{2 . 6 5 e + 5}$ & $\mathbf{1 . 3 3 e}+\mathbf{5}$ & $\mathbf{6 . 6 3 e}+\mathbf{4}$ & $\mathbf{3 . 3 1 e + 4}$ \\
\hline
\end{tabular}

Table 2. Errors of cubic spline on piecewise-uniform grid

\begin{tabular}{lllllll}
\hline \multicolumn{1}{c}{$\mathbf{N}$} & $\mathbf{1 6}$ & $\mathbf{3 2}$ & $\mathbf{6 4}$ & $\mathbf{1 2 8}$ & $\mathbf{2 5 6}$ & $\mathbf{5 1 2}$ \\
$\boldsymbol{\varepsilon}$ & & & & & & \\
\hline $\mathbf{1}$ & $2.82 \mathrm{e}-7$ & $1.76 \mathrm{e}-8$ & $1.16 \mathrm{e}-9$ & $1.02 \mathrm{e}-10$ & $4.30 \mathrm{e}-11$ & $\mathbf{2 . 6 8 e - 1 3}$ \\
$\mathbf{1 0 e}-\mathbf{1}$ & $3.43 \mathrm{e}-4$ & $2.33 \mathrm{e}-5$ & $1.51 \mathrm{e}-6$ & $9.58 \mathrm{e}-8$ & $6.03 \mathrm{e}-9$ & $\mathbf{4 . 1 1 e - 1 0}$ \\
$\mathbf{1 0 e}-2$ & $6.43 \mathrm{e}-3$ & $1.18 \mathrm{e}-3$ & $1.70 \mathrm{e}-4$ & $2.07 \mathrm{e}-5$ & $2.27 \mathrm{e}-6$ & $\mathbf{2 . 3 1 e - 7}$ \\
$\mathbf{1 0 e}-3$ & $6.43 \mathrm{e}-3$ & $1.18 \mathrm{e}-3$ & $1.70 \mathrm{e}-4$ & $2.07 \mathrm{e}-5$ & $2.27 \mathrm{e}-6$ & $\mathbf{2 . 3 1 e - 7}$ \\
$\mathbf{1 0 e}-4$ & $6.43 \mathrm{e}-3$ & $1.18 \mathrm{e}-3$ & $1.70 \mathrm{e}-4$ & $2.07 \mathrm{e}-5$ & $2.27 \mathrm{e}-6$ & $\mathbf{2 . 3 1 e - 7}$ \\
$\mathbf{1 0 e}-\mathbf{5}$ & $4.47 \mathrm{e}-2$ & $1.25 \mathrm{e}-3$ & $1.70 \mathrm{e}-4$ & $2.07 \mathrm{e}-5$ & $2.27 \mathrm{e}-6$ & $\mathbf{2 . 3 1 e - 7}$ \\
$\mathbf{1 0 e}-\mathbf{6}$ & $4.47 \mathrm{e}-1$ & $1.25 \mathrm{e}-2$ & $3.62 \mathrm{e}-4$ & $2.07 \mathrm{e}-5$ & $2.27 \mathrm{e}-6$ & $\mathbf{2 . 3 1 e - 7}$ \\
$\mathbf{1 0 e}-\mathbf{7}$ & 4.47 & $1.25 \mathrm{e}-1$ & $3.62 \mathrm{e}-3$ & $1.07 \mathrm{e}-4$ & $3.24 \mathrm{e}-6$ & $\mathbf{2 . 3 1 e - 7}$ \\
\hline $\mathbf{1 0 e}-\mathbf{8}$ & $\mathbf{4 . 4 7}$ & $\mathbf{1 . 2 5}$ & $\mathbf{3 . 6 2 e}-\mathbf{2}$ & $\mathbf{1 . 0 7 e - 3}$ & $\mathbf{3 . 2 4 e}-\mathbf{5}$ & $\mathbf{9 . 9 2 e - 7}$ \\
\hline
\end{tabular}

Table 3. Errors of modified cubic spline on piecewise-uniform grid

\begin{tabular}{lllllll}
\hline \multicolumn{1}{c}{$\mathbf{N}$} & $\mathbf{1 6}$ & $\mathbf{3 2}$ & $\mathbf{6 4}$ & $\mathbf{1 2 8}$ & $\mathbf{2 5 6}$ & $\mathbf{5 1 2}$ \\
$\boldsymbol{\varepsilon}$ & & & & & & \\
\hline $\mathbf{1}$ & $3.1 \mathrm{e}-7$ & $2.0 \mathrm{e}-8$ & $1.3 \mathrm{e}-9$ & $1.1 \mathrm{e}-10$ & $4.9 \mathrm{e}-12$ & $\mathbf{3 . 1 e}-\mathbf{1 3}$ \\
$\mathbf{1 0 e}-\mathbf{1}$ & $3.4 \mathrm{e}-4$ & $2.3 \mathrm{e}-5$ & $1.5 \mathrm{e}-6$ & $9.6 \mathrm{e}-8$ & $6.03 \mathrm{e}-9$ & $\mathbf{4 . 1 e}-10$ \\
$\mathbf{1 0 e}-2$ & $6.43 \mathrm{e}-3$ & $1.2 \mathrm{e}-3$ & $1.7 \mathrm{e}-4$ & $2.1 \mathrm{e}-5$ & $2.3 \mathrm{e}-6$ & $\mathbf{2 . 3 e}-7$ \\
$\mathbf{1 0 e}-\mathbf{3}$ & $6.43 \mathrm{e}-3$ & $1.2 \mathrm{e}-3$ & $1.70 \mathrm{e}-4$ & $2.07 \mathrm{e}-5$ & $2.3 \mathrm{e}-6$ & $\mathbf{2 . 3 e}-\mathbf{7}$ \\
$\mathbf{1 0 e}-\mathbf{4}$ & $6.43 \mathrm{e}-3$ & $1.2 \mathrm{e}-3$ & $1.70 \mathrm{e}-4$ & $2.07 \mathrm{e}-5$ & $2.3 \mathrm{e}-6$ & $\mathbf{2 . 3 e}-\mathbf{7}$ \\
$\mathbf{1 0 e}-\mathbf{5}$ & $6.43 \mathrm{e}-3$ & $1.2 \mathrm{e}-3$ & $1.70 \mathrm{e}-4$ & $2.07 \mathrm{e}-5$ & $2.3 \mathrm{e}-6$ & $\mathbf{2 . 3 e}-\mathbf{7}$ \\
$\mathbf{1 0 e}-\mathbf{6}$ & $6.43 \mathrm{e}-3$ & $1.2 \mathrm{e}-3$ & $1.70 \mathrm{e}-4$ & $2.07 \mathrm{e}-5$ & $2.3 \mathrm{e}-6$ & $\mathbf{2 . 3 e}-\mathbf{7}$ \\
$\mathbf{1 0 e}-\mathbf{7}$ & $6.43 \mathrm{e}-3$ & $1.2 \mathrm{e}-3$ & $1.70 \mathrm{e}-4$ & $2.07 \mathrm{e}-5$ & $2.3 \mathrm{e}-6$ & $\mathbf{2 . 3 e}-\mathbf{7}$ \\
\hline $\mathbf{1 0 e}-\mathbf{8}$ & $\mathbf{6 . 4 3 e - 3}$ & $\mathbf{1 . 2 e}-\mathbf{3}$ & $\mathbf{1 . 7 0 e}-\mathbf{4}$ & $\mathbf{2 . 0 7 e - 5}$ & $\mathbf{2 . 3 e}-\mathbf{6}$ & $\mathbf{2 . 3 e - 7}$ \\
\hline
\end{tabular}




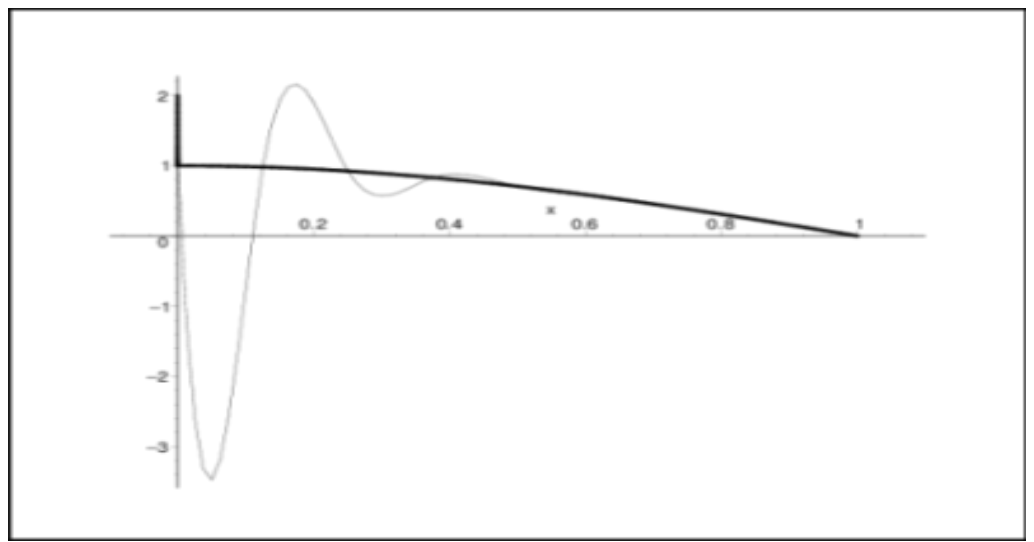

Fig. 1. Graphs of function (8) and of its interpolation spline on the piecewise uniform grid at the $[0,1]$ interval.

\section{Acknowledgement}

The work is supported by Russian Foundation of Basic Researches under Grant 1501-06584.

\section{References}

1. Ahlberg JH. The theory of splines and their applications. New York: Academic Press, 1967; $284 \mathrm{p}$.

2. Zav'yalov YuS, Kvasov BI, Miroshnichenko VL. Methods of Spline Functions. Moscow: Nauka, 1980; 352 p. [in Russian]

3. Zadorin AI. Method of interpolation for a boundary layer problem. Sib. J. of Numer Math., 2007; 10(3): 267-275. [in Russian]

4. Zadorin AI, Guryanova MV. Analogue of a cubic spline for a function with a boundary layer component. Proceedings of the fifth conference on finite difference methods: Theory and Applications, 2010. Rousse: Rousse University, 2011: 166-173.

5. Zmatrakov NL. Convergence of an interpolation process for parabolic and cubic splines. Trudy Mat. Inst. Steklov, 1975; 138: 71-93.

6. Zmatrakov NL. A necessary condition for convergence of interpolating parabolic and cubic splines. Mat. Zametki, 1976; 19(2): 100-107. DOI: 10.1007/BF01098740.

7. Shishkin GI. Discrete Approximations of Singularly Perturbed Elliptic and Parabolic Equations. Ekaterinburg: Russian Academy of Sciences, Ural Branch, 1992; 233 p. [in Russian] 